\title{
Preparation and degradation behaviour of Bi-cuprate superconductors
}

\author{
BALVINDER GOGIA, SUBHASH C KASHYAP, D K PANDYA \\ and K L CHOPRA \\ Physics Department. Indian Institute of Technology, New Delhi 110016, India
}

\begin{abstract}
The role of preparation conditions and the effect of addition of $\mathrm{Pb}$ in $\mathrm{Bi}-\mathrm{Sr}-\mathrm{Ca}-$ $\mathrm{Cu}-\mathrm{O}$ (BSCCO) superconductor, on the $T_{c}, J_{c}$ and grain orientation have been studied. Calcination at a temperature higher than the melting point of $\mathrm{Bi}_{2} \mathrm{O}_{3}$ after the prereaction at $800^{\circ} \mathrm{C}$ leads to formation of $($-axis oriented nearly single phase material. The presence of lead yields a nearly high $T_{c}$ phase (2223) exhibiting a maximum transition temperature $T_{c}=110 \mathrm{~K}$ and $\Delta T_{c}=2 \mathrm{~K}$. The addition of lead and grain orientation together result in an increase in $J_{c}$ by nearly two orders of magnitude. The degradation behaviour was studied in terms of changes in $T_{c}, J_{c}$ and structure on exposure to atmosphere up to 250 days. Samples with $0.6 \mathrm{~Pb}$ were found to be superconducting with $T_{c}=97 \mathrm{~K}$ even after 250 days. The high $T$, phase was stable against degradation. Our studies indicate that the degradation of leaded BSCCO is a surface phenomenon rather than bulk phenomenon.
\end{abstract}

Keywords. High temperature superconductors: leaded BSCCO superconductors; ('-axis orientation; degradation behaviour.

\section{Introduction}

The discovery of Bi-(Michel et al 1987: Maeda et al 1988) and Tl- (Sheng and Hermann 1988) cuprate superconductors has attracted the attention of many research groups due to their higher zero resistance temperature, $T_{c}(R=0)$ or simply $T_{c}$. The Bi-cuprate system, in general, has three different phases corresponding to $T_{c}$ close to 20,80 and $110 \mathrm{~K}$. Numerous studies have been made to synthesize and stabilize the pure high $T_{c}$ $(110 \mathrm{~K})$ phase by varying the processing parameters like cation non-stoichiometry (Koyama et al 1988), heat treatment conditions (Tarascon et al 1988), cooling rate (Kikuchi et al 1989) and by the addition of $\mathrm{Pb}$ (Sunshine et al 1988; Statt et al 1988) to the $\mathrm{Bi}-\mathrm{Sr}-\mathrm{Ca}-\mathrm{Cu}-\mathrm{O}$ (BSCCO) system. However, the highest value of $T_{\mathrm{c}}$ was achieved only when sintering was done for long durations (Chavira et al 1988; Kim et al 1989) up to $200-300 \mathrm{hr}$. Besides, critical current density, $J_{c}$, of these materials is still very low as compared to the YBCO superconductor. One of the reasons is the random growth of the plate-like grains of the high $T_{c}$ phase.

The BSCCO superconductors need to be densified and the grains oriented so that their $J_{c}$ is increased. Some special techniques like intermediate cold pressing (Asano et al 1988; Ito 1989), hot pressing (Murayama et al 1988), low oxygen partial pressure during sintering (Endo et al 1988) etc. have been tried to obtain $c$-axis oriented samples having higher $J_{i .}$. These techniques are, however, quite elaborate and complicate the processing steps. We have tried to achieve the same by simply changing the reaction process conditions.

The conversion of 2223 phase into low $T_{c}$ phase is one of the major problems of environmental stability thus lowering the $T_{c}$ and $J_{c}$. We have studied this stability in the bulk samples for over 250 days after their preparation. 


\section{Experimental}

The BSCCO samples were prepared using cationic ratio of $\mathrm{Bi}: \mathrm{Pb}: \mathrm{Sr}: \mathrm{Ca}: \mathrm{Cu}$ as $2.0: x: 2.0: 2.0: 3.1(0.0 \leqslant x \leqslant 0.8)$. In the first set of samples, called batch $\mathrm{A}$, the powders were calcined at $800^{\circ} \mathrm{C}$. The calcined mass was again heated at $800^{\circ} \mathrm{C}$ for $20 \mathrm{hr}$. In the second set of samples only two compositions, $\mathrm{Bi}_{2} \mathrm{~Pb}_{0.6} \mathrm{Sr}_{2} \mathrm{Ca}_{2} \mathrm{Cu}_{3.1} \mathrm{O}_{y}$ (batch $\mathrm{B}$ ) and $\mathrm{Bi}_{2} \mathrm{Sr}_{2} \mathrm{Ca}_{2} \mathrm{Cu}_{3.1} \mathrm{O}_{y}$ (batch $\mathrm{C}$ ) were used. In this set too, the well-ground powders were pre-reacted at $800^{\circ} \mathrm{C}$. The calcined mass was again ground and heated at $800^{\circ} \mathrm{C}$ for $2 \mathrm{hr}$ followed by a slow increase in temperature to $820^{\circ} \mathrm{C}\left(\sim\right.$ melting point of $\mathrm{Bi}_{2} \mathrm{O}_{3}$ ), which was maintained for $3 \mathrm{hr}$. Finally the temperature was raised to $840^{\circ} \mathrm{C}$ and maintained for $12 \mathrm{hr}$. The calcined mass so obtained was reground. The pellets, made at a pressure of 6 ton $/ \mathrm{cm}^{2}$, were first sintered in air at a temperature $3-4^{\circ} \mathrm{C}$ lower than their melting point for $20 \mathrm{~min}$. They were then kept at a temperature $10^{\circ} \mathrm{C}$ lower than their melting point for different durations viz 75 to $125 \mathrm{hr}$. The pellets were cooled in $\mathrm{O}_{2}$ till $500^{\circ} \mathrm{C}$ where they were kept for $24 \mathrm{hr}$ before cooling to room temperature.

Rectangular bars $(18 \mathrm{~mm} \times 2 \mathrm{~mm} \times 1 \mathrm{~mm})$ were cut from sintered pellets for resistivity and $J_{c}$ measurements. Resistivity was measured in the temperature range of $300-15 \mathrm{~K}$ by the d.c. four-probe method using indium contacts on silver electrodes. All $J_{c}$ measurements were done at $77 \mathrm{~K}$. $J_{c}$ was calculated using the usual criteria of $1 \mu \mathrm{V} / \mathrm{cm}$. For structural analysis $\mathrm{X}$-ray diffraction data were obtained by employing Rigaku's RU $200 \mathrm{~B}$ X-ray diffractometer using $\mathrm{CuK}_{\alpha}$ radiation.

\section{Results and discussion}

Figure 1 shows the variation of $T_{c}$ with $\mathrm{Pb}$ concentration in the range $x=0$ to 0.8 (samples $\mathrm{A} 1$ to $\mathrm{A} 5$ ). $T_{c}$ maximizes at $105 \mathrm{~K}$ (sample A4) for $x=0.6$. All the leaded samples showed single-step transition. The critical current density (figure 2) also showed similar variation as $T_{c}$ and maximizes at $15 \mathrm{~A} / \mathrm{cm}^{2}$ for sample A4. Since $x=0.6$ was the most appropriate $\mathrm{Pb}$ concentration from the point of view of both $T_{c}$ and $J_{c}$, this concentration was selected for further investigations. The samples B1, B2 and B3

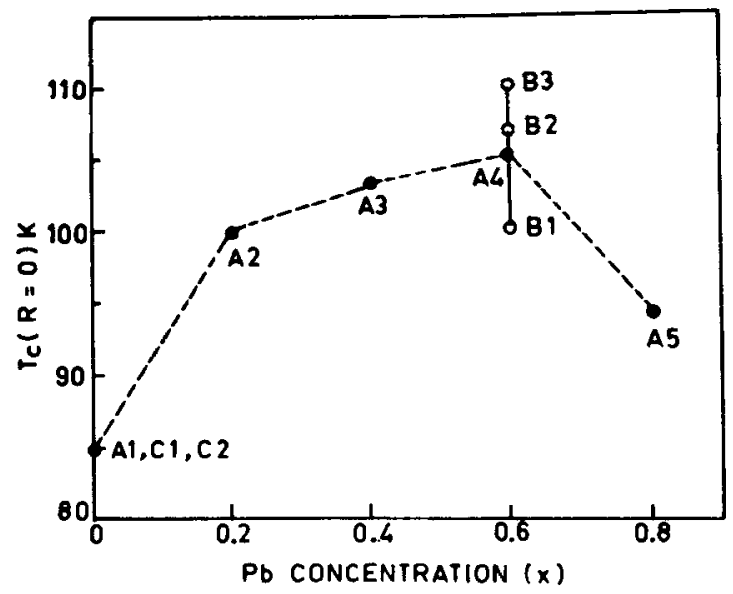

Figure 1. Variation of $T_{c}$ with $\mathrm{Pb}$ concentration (samples $\mathrm{A} 1$ to $\mathrm{A} 5$ ) and sintering time (B1 to B3 and C1, C2). 


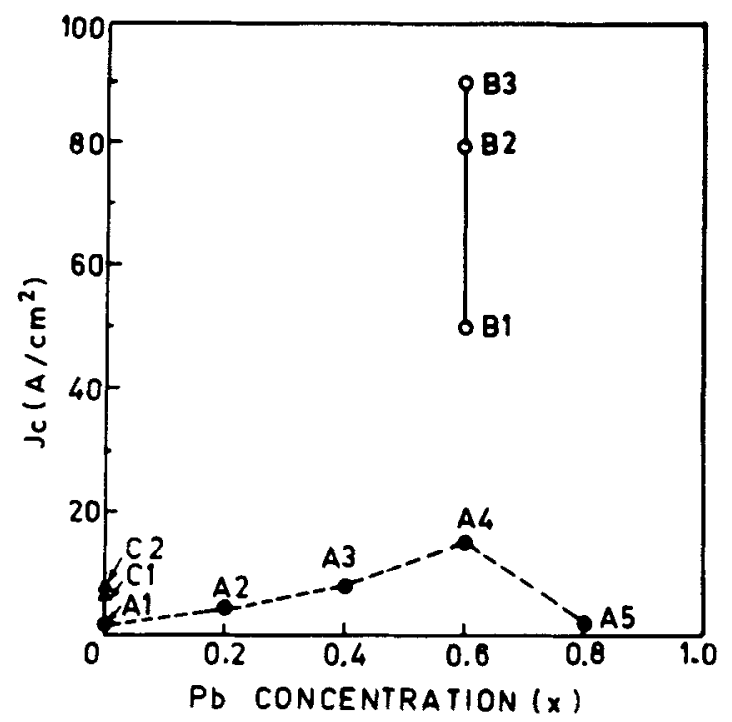

Figure 2. Variation of $J$, with $\mathrm{Pb}$ concentration (samples $\mathrm{A} 1$ to $\mathrm{A} 5$ ) and sintering time (B1 to $\mathrm{B} 3$ and $\mathrm{C} 1, \mathrm{C} 2)$

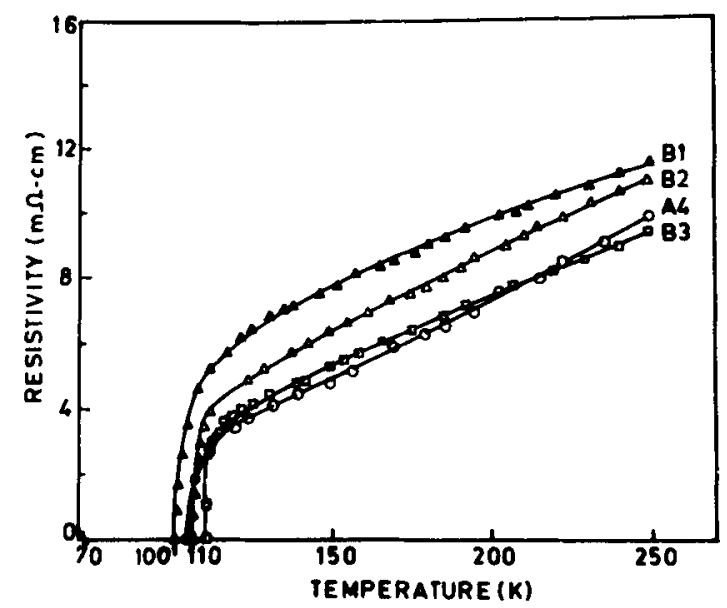

Figure 3. Temperature dependence of resistivity of samples B1, B2, B3 and A4.

were sintered for 75,100 and $125 \mathrm{hr}$ respectively. For comparison we include the results of lead-free samples, $\mathrm{Cl}$ and $\mathrm{C} 2$, sintered for 100 and $125 \mathrm{hr}$, respectively.

Whereas in lead-free samples (batch C) no improvement in $T_{c}(=85 \mathrm{~K}$ ) was observed, the transition width and $J_{c}$ improved over batch $\mathrm{A}$. As shown in figure $2 J_{c}$ increased from $1 \mathrm{~A} / \mathrm{cm}^{2}$ (sample $\mathrm{A} 1$ ) to $6 \mathrm{~A} / \mathrm{cm}^{2}$ and $7 \mathrm{~A} / \mathrm{cm}^{2}$ for samples $\mathrm{Cl}$ and $\mathrm{C} 2$, respectively. In comparison, batch B samples showed an increase in both $T_{c}$ and $J_{c}$. As shown in figure 3, all these samples exhibited a single-step transition with a $T_{c}$ of 100,107 and $110 \mathrm{~K}$, respectively and the transition width narrowing down from $10 \mathrm{~K}$ for $\mathrm{B} 1$ to $2 \mathrm{~K}$ for B3. Associated with an improvement in $T_{c}$, an increase in $J_{c}$ was also observed. The samples B1, B2 and B3 had a $J_{c}$ of 50,80 and $90 \mathrm{~A} / \mathrm{cm}^{2}$ (figure 2 ) respectively. It is thus 


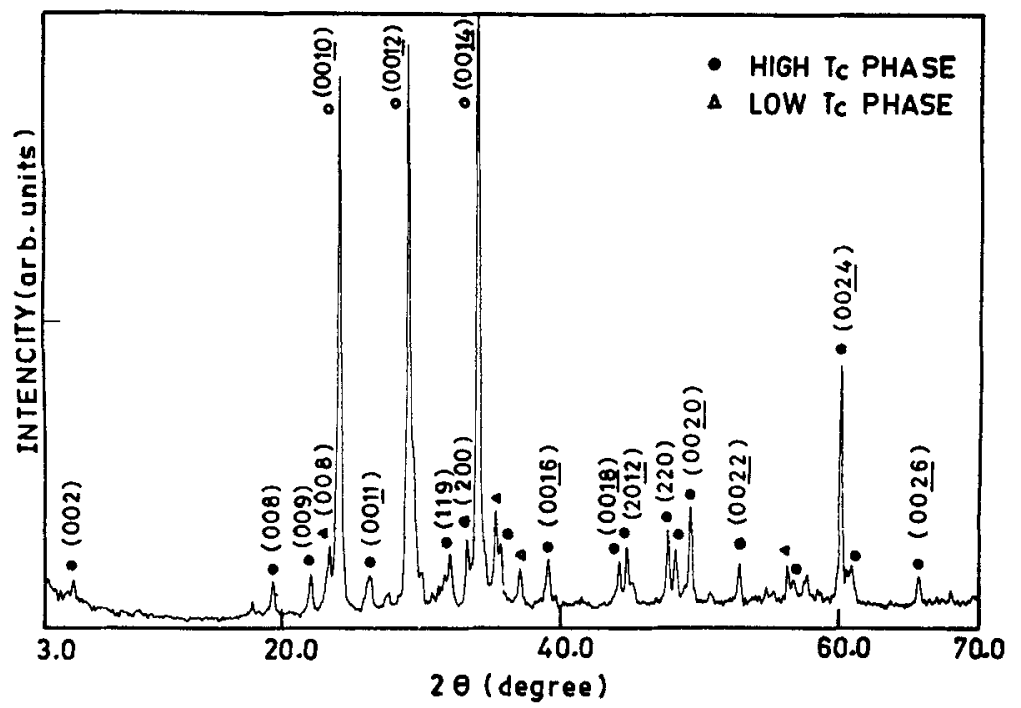

Figure 4. X-ray diffraction pattern of $c$-axis oriented sample B3. ( $)$ High $T_{\mathrm{c}}$ phase, $(\Delta)$ low $T_{c}$ phase.

observed that the modification of the preparation condition leads to improvement in $J_{c}$ by about an order of magnitude. This, in association with the $\mathrm{Pb}$ addition, improves $J_{c}$ by two orders of magnitude.

All the samples in batch A showed an orthorhombic structure (Gogia et al 1990) with coexistence of both - low and high $T_{c}$ phases. However, no significant orientation effect was observed in these samples. In contrast, the samples in batch B showed strong orientation effects. The $c$-axis is oriented perpendicular to the sample surface as seen from figure 4 showing X-ray diffraction pattern of sample B3. With increase in sintering time, the extent of orientation increased and the low $T_{c}$ phase content decreased. Leadfree samples (batch $C$ ) which exhibited predominantly low $T_{c}$ phase also showed the same $c-$ axis orientation effect. Therefore, the improvement in $J_{c}$ in our batch B and C samples may be attributed to grain orientation (due to prereaction) and addition of lead (due to evolution of single 2223 phase).

It is clear from both electrical and structural properties of BSCCO superconductors that calcination at temperatures higher than the melting point of $\mathrm{Bi}_{2} \mathrm{O}_{3}$ after prereaction at $800 \mathrm{C}$ helps in the formation of c-axis oriented nearly single high $T_{c}$ phase thus improving $J_{c}$ as well as $T_{c}$ without requiring any special technique.

The environmental degradation behaviour of BSCCO superconductors was studied in terms of change in $T_{\mathfrak{c}}, J_{\mathfrak{c}}$ and structure on exposure of these superconductors to atmosphere. The $T_{c}$ and $J_{c}$ of the two samples, one of lower $T_{c}$ (A1) and the other of higher $T_{c}$ (A4), were measured on 1st, 21st, 51st, 111 th, 150th and 250th day of their preparation. X-ray patterns were also recorded on the same days. The $T_{c}$ and $J_{c}$ both decreased on exposure of samples to atmosphere. In case of $\mathrm{Al}, T_{c}$ continuously decreased from 85 to $65 \mathrm{~K}$ till 250 days after its preparation. In leaded superconductor (sample A4), $T_{c}$ had gone down from 105 to $98 \mathrm{~K}$ at the end of 111 days after which it became almost constant at $97 \mathrm{~K}$ till 250 days. This suggests a remarkable improvement in atmospheric stability of leaded BSCCO superconductor. Changes in $J_{c}$ were also minimal for A4. The values of $T_{c}$ and $J_{c}$ are presented in table 1 . 
Table 1. Variation of $T_{c}$ and $J_{c}$ of samples $\mathrm{A} 1$ and $\mathrm{A} 4$ with exposure time to atmosphere.

\begin{tabular}{rcccc}
\hline & \multicolumn{2}{c}{$\begin{array}{c}\mathrm{A} 1 \\
(\mathrm{x}=0.0)\end{array}$} & \multicolumn{2}{c}{$\begin{array}{c}\mathrm{A} 4 \\
(\mathrm{x}=0.6)\end{array}$} \\
\cline { 2 - 5 } Day & $\begin{array}{c}T_{c} \\
(\mathrm{~K})\end{array}$ & $\begin{array}{c}J_{c} \\
\left(\mathrm{~A} / \mathrm{cm}^{2}\right)\end{array}$ & $\begin{array}{c}T_{c} \\
(\mathrm{~K})\end{array}$ & $\begin{array}{c}J_{c} \\
\left(\mathrm{~A} / \mathrm{cm}^{2}\right)\end{array}$ \\
\hline 1 & 85 & 1.0 & 105 & 15 \\
21 & 80 & 0.4 & 103 & 14 \\
51 & 76 & - & 101 & 13 \\
111 & 71 & - & 98 & 12 \\
150 & 68 & - & 97.5 & 11 \\
250 & 65 & - & 97 & 10 \\
\hline
\end{tabular}

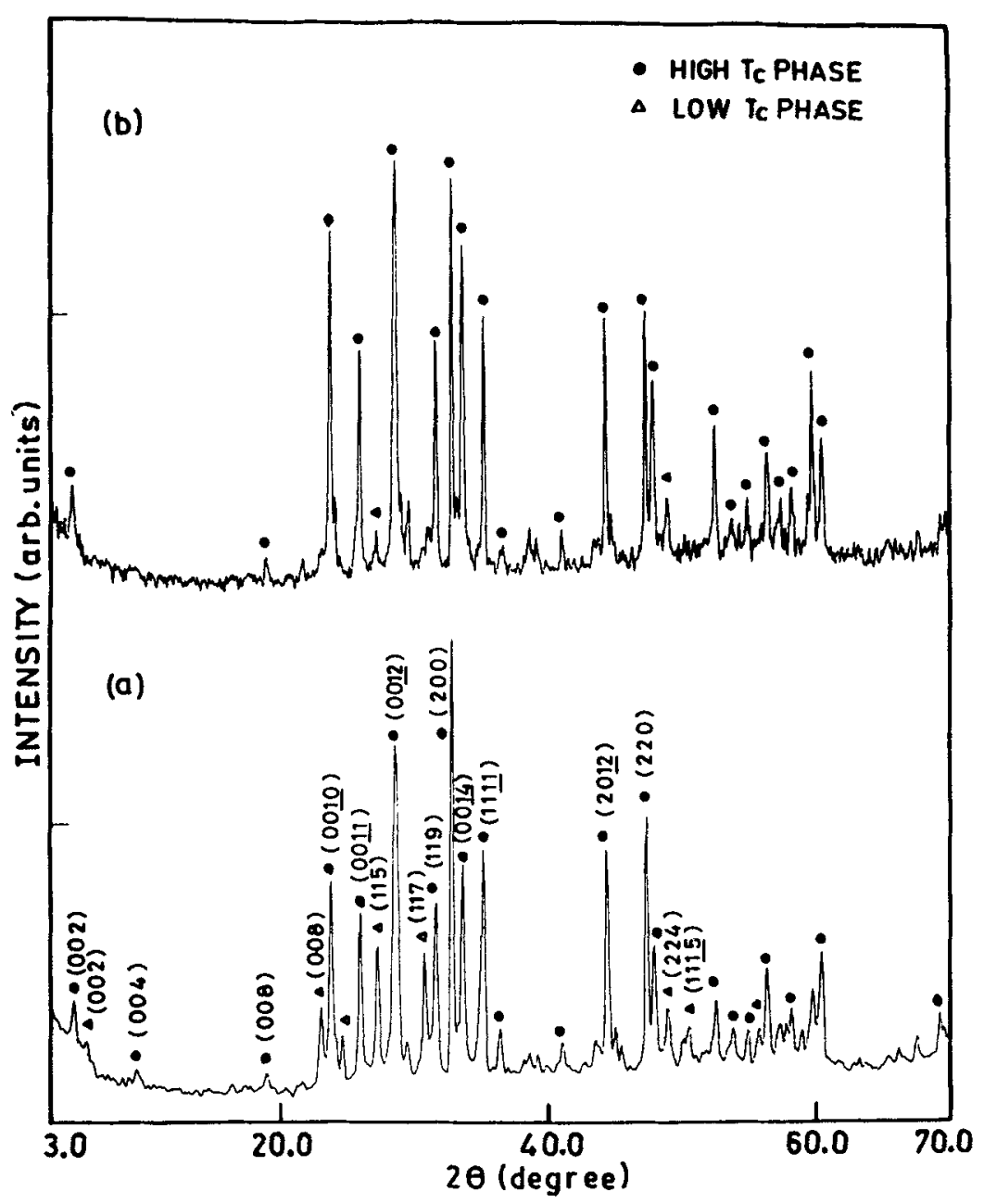

Figure 5. X-ray diffraction patterns of sample A4 recorded on (a) 1st day of its preparation and (b) after 250 days of preparation. 
Structural analysis of A4 showed some striking features. This sample initially had both low and high $T_{c}$ phases (figure $5 \mathrm{a}$ ) which were clearly discernible. But after 50 days a predominantly high $T_{c}$ phase was observed with almost sudden decrease in intensities of low $T_{c}$ phase peaks. Beyond this period no further change (figure $5 \mathrm{~b}$ ) was observed. It looked as if lead had completely stabilized the high $T_{c}$ phase. In sharp contrast to A4, sample A1, which initially had a few low intensity peaks of high $T_{c}$ phase along with dominant peaks corresponding to low $T_{c}$ phase, showed almost complete disappearance of high $T_{c}$ phase on exposure of these samples to atmosphere. This conforms with the vanishing of the step in resistivity measurements corresponding to the high $T_{c}$ phase. It is clear that low $T_{c}$ phase is quite stable in the $\mathrm{Pb}$-free sample but not the high $T_{c}$ phase. However, once the high $T_{c}$ phase is stabilized with the addition of $\mathrm{Pb}$, it does not degrade structurally on exposure to atmosphere. It is suggested that the observed decrease in $T_{c}$ and $j_{c}$ of leaded BSCCO superconductor when exposed to atmosphere is not a bulk degradation phenomenon but may be due to surface degradation as a result of carbonate/hydroxide formation on the surface.

\section{References}

Asano T, Tanaka Y, Fukutomi M, Jikihara K, Machida J and Maeda H 1988 Jpn. J. Appl. Phys. 27 L1652 Chavira E, Escudero D, Riós-Jara and Leon L M 1988 Phys. Rev. B38 9272

Endo U, Koyama S and Kawai T 1988 Jpn. J. Appl. Phys. 27 L1476

Gogia B, Kashyap S C, Pandya D K and Chopra K L 1989 Solid State Commun. 73573 Ito A 1989 Jpn. J. Appl. Phys. 28 L380

Kikuchi A, Matsude M, Takata M, Ishii M, Yamashita T and Koinuma H 1989 Jpn. J. Appl. Phys. 28 L371

Kim C J, Rhee C K, Lee H G, Lee C T, Kang S J-L and Won D Y $1989 J$ pn. J. Appl. Phys. 28 L45

Koyama S. Endo U and Kawai T 1988 Jpn. J. Appl. Phys. 27 L1861

Maeda H, Tanaka Y, Fukutomi M and Asano T 1988 Jpn. J. Appl. Phys. 27 L209

Michel C, Herview M. Borel M M, Grandin A, Deslandes I, Provost J and Raveau B 1987 Z. Phys. B. 68421

Murayama N, Sudo E. Awano M, Kani K and Torii Y 1988 Jpn. J. Appl. Phys. 27 L1856

Sheng $Z Z$ and Hermann A M 1988 Nature (London) 33255 and 138

Statt B W, Wang Z, Lee M J G, Yakhmi J V, deCamargo P C, Major J F and Rutler J W 1988 Physica C156 251

Sunshine S A et al 1988 Phys. Rev. B38 893

Tarascon J M, Le Page Y, Barboux P, Bagley B G, Greene L H, McKinnon W R, Hull G W, Giroud M and Hwang D M 1988 Phys. Rev. B37 9382 